\title{
Synthesis and Characterization of Bovine Serum Albumin-Conjugated Copper Sulfide Nanocomposites
}

\author{
Peng Huang, Zhiming Li, Hengyao Hu, and Daxiang Cui \\ Department of Bio-Nano Science and Engineering, Key Laboratory for Thin Film and Microfabrication Technology of Ministry of \\ Education, National Key Laboratory of Micro/Nano Fabrication Technology, Research Institute of Micro/Nano Science and Technology, \\ Shanghai Jiao Tong University, Shanghai 200240, China
}

Correspondence should be addressed to Daxiang Cui, dxcui@sjtu.edu.cn

Received 27 September 2009; Revised 31 December 2009; Accepted 2 April 2010

Academic Editor: Maryam Tabrizian

Copyright (C) 2010 Peng Huang et al. This is an open access article distributed under the Creative Commons Attribution License, which permits unrestricted use, distribution, and reproduction in any medium, provided the original work is properly cited.

\begin{abstract}
A simple biomolecule-assisted solution route was developed to synthesize Bovine Serum Albumin-conjugated copper sulfide (CuS/BSA) nanocomposites, directly using copper salts and thioacetamide (TAA) as the starting materials with a zwitterionic surfactant Bovine Serum Albumin (BSA) as foaming and stabilizing agent. The CuS/BSA nanocomposites have been characterized by UV, TEM, Zeta, DLS, XRD, and FTIR. The results indicate that the as-prepared CuS/BSA nanocomposites are approximate sphere with a size distribution from 10 to $35 \mathrm{~nm}$ in diameter and good dispersibility, depending highly on concentration of BSA concentration. These protein-assisted synthesized nanocomposites have a great potential application in biomedical engineering and microelectronics.
\end{abstract}

\section{Introduction}

Chalcogenide semiconductors have attracted considerable attention due to their nonlinear properties, photoluminescent properties and other useful physical and chemical properties [1-5]. However, controllable synthesis of these semiconductor nanomaterials is still a challengeable task. In recent years, copper sulfide $(\mathrm{CuS})$ has been widely used in many fields such as pigment, catalyzer, optical filter, solar cell, and ion conduction [6]. Therefore, CuS nanocrystallites with different morphologies such as nanoparticles, nanodisk, nanorods, nanowires, and nanotubes have been synthesized by considerable methods, including sonochemical methods [1], microwave-assisted methods [7], electrosynthesis [8], hydrothermal methods [9], $\gamma$-irradiation routes [10], microemulsion-directed routes [11], solvothermal process [12], solid-state reactions [13], mechanochemical route [14], and chemical vapor deposition (CVD) [15]. Among these methods, most of them need special instruments, high temperature, high pressure, inert gas environment, and long reaction time. So how to use the simplest routes and the cheapest raw materials to fabricate high-quality $\mathrm{CuS}$ nanomaterials has become a hotspot.
In the past decade, many studies show that biological macromolecules, such as amino acids, proteins, DNA and RNA, are capable of controlling nanomaterials nucleation and growth to various different degrees [16]. The obtained biomolecules-conjugated nanomaterials are bioactive and biocompatible, which could provide bioactive functionalities throughout the nanocrystal surface for further biological interactions or couplings and then be used in life sciences for luminescence tagging, drug delivery, and many other aspects [16-18]. Bovine Serum Albumin (BSA), one of the most widely studied proteins [19], has been frequently adopted to synthesize various nanocrystals. Singh et al. have prepared protein capped Ag [20,21], Au [20], Pt [21], Ag-Au [20], Ag-Pt [21] nanomaterials in aqueous BSA foam and study their cytocompatibility against human gingival fibroblasts. Yang et al. have successfully fabricated BSA-ponjugated $\mathrm{Ag}_{2} \mathrm{~S}$ Nanorods [22, 23] and HgS nanoparticles [24], and discussed their formation mechanism. Our group firstly reported a simple, environment-friendly, controllable synthetic method of CuSe nanosnakes at room temperature using BSA as foaming agent [25]. All these results suggest a possible biomimetic role for BSA as "soft template" in materials chemistry. 
Herein, we firstly reported a convenient, simple, nontoxic, and environment-friendly method to obtain $\mathrm{CuS}$ nanoparticles in the BSA solution. Our results showed that the synthesized CuS/BSA nanocomposites have controllable sizes and sphere shapes, which lay foundation for investigating further application in different fields.

\section{Experimental}

2.1. Materials. Copper nitrate $\left(\mathrm{Cu}\left(\mathrm{NO}_{3}\right)_{2}\right)$ and thioacetamide (TAA) were A.R. reagents from Sinopharm Company, China. BSA was of electrophoretic purity, purchased from Xiamen Sanland Chemicals Company Limited, China. The average molecular weight of it is about $68,000 \mathrm{Da}$. All the abovementioned chemicals were used without any further purification.

2.2. Synthesis of CuS/BSA Nanocomposites. In a typical process, $5 \mathrm{~mL}$ of $25 \mathrm{mMCu}\left(\mathrm{NO}_{3}\right)_{2}$ aqueous solution and $10 \mathrm{~mL}$ of $1 \sim 4 \mathrm{mg} / \mathrm{mL}$ BSA aqueous solution were mixed with vigorous stirring at room temperature. The mixed solution of the BSA-Cu${ }^{2+}$ emulsion was kept static under nitrogen protection for 2 hours. Then $5 \mathrm{~mL}$ of $25 \mathrm{mM}$ TAA aqueous solution were added. Immediately after TAA addition, the solution changed to black, indicating the formation of colloidal $\mathrm{CuS}$ particles. The mixed reaction solution was again kept static under ambient conditions for 96 hours and then separated by high speed centrifuging at $15000 \mathrm{rpm}$. The collected black solidstate product was washed with double distilled water and ethanol for three times and dried in a vacuum at room temperature for 24 hours.

2.3. Characterization of CuS/BSA Nanocomposites. UV-vis spectra were measured at $20^{\circ} \mathrm{C}$ with a Shimadzu UV2450 UV-visible spectrophotometer equipped with a 10$\mathrm{mm}$ quartz cell. The size and morphology of nanoparticles were obtained by using a JEOL JEM-2010 transmission electron microscope, operating at an accelerating voltage of $200 \mathrm{kV}$. Zeta potential and Particle size were measured with NICOMP 380ZLS Zeta potential/Particle sizer. These samples were characterized by using Rigaku D/max-2200/PC $\mathrm{X}$-ray diffractometer with monochromated $\mathrm{CuKa}$ radiation operated at $40 \mathrm{kV}$ and $100 \mathrm{~mA}$. The Fourier transform infrared (FTIR) spectra were recorded on a Perkin Elmer Paragon-1000 FTIR Spectrometer.

\section{Results and Discussion}

3.1. UV-Vis Measurement. Figure 1 shows the UV spectra of pure BSA, BSA-Cu ${ }^{2+}$, and BSA-CuS in the $190-400 \mathrm{~nm}$ wavelength range. The spectrum of pure BSA showed the special absorption peak at $280 \mathrm{~nm}$. Curve (b) was the spectrum of BSA-Cu ${ }^{2+}$ complex, showing no obvious shift of absorption peak at $280 \mathrm{~nm}$ and significantly enhancement of absorption before $280 \mathrm{~nm}$, which is probably because the BSA protein can provide multiple binding sites for $\mathrm{Cu}^{2+}, \mathrm{Cu}^{2+}$ can react with BSA, and the binding sites may include the $-\mathrm{NH},-\mathrm{SH},-\mathrm{COOH}$, and $-\mathrm{OH}$ groups. The

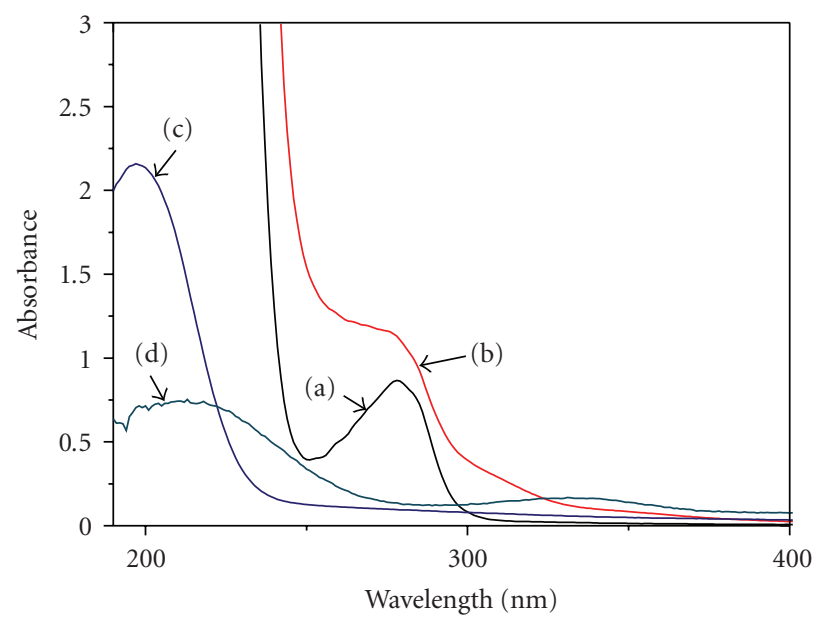
(a) BSA
(b) $\mathrm{BSA}-\mathrm{Cu}^{2+}$
(c) BSA-CuS
(d) $\mathrm{CuS}$

FIGURE 1: UV spectra of (a) BSA, (b) BSA-Cu${ }^{2+}$, (c) BSA-CuS, and (d) CuS.

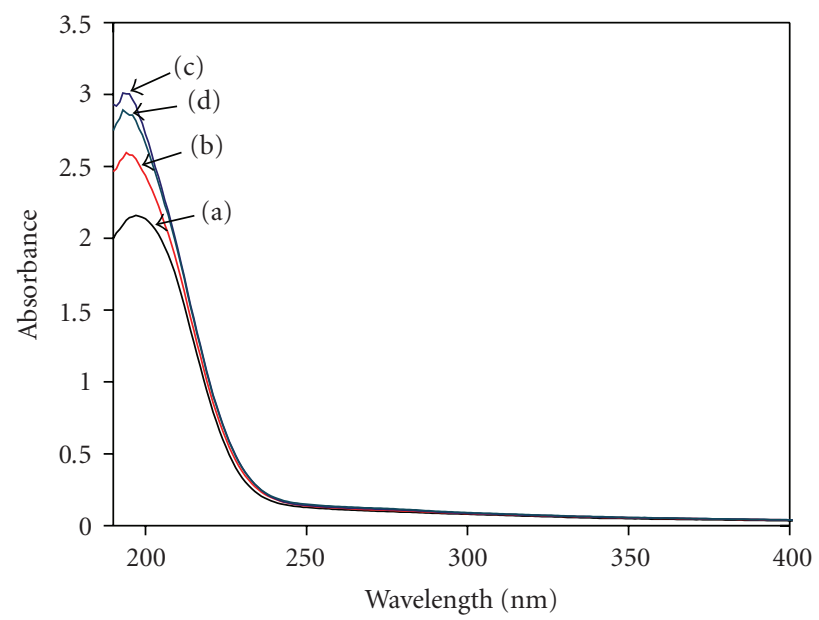
(a) $1 \mathrm{mg} / \mathrm{mL}$
(b) $2 \mathrm{mg} / \mathrm{mL}$
(c) $3 \mathrm{mg} / \mathrm{mL}$
(d) $4 \mathrm{mg} / \mathrm{mL}$

FIGURE 2: UV spectra of CuS/BSA nanocomposites synthesized in different concentration BSA solution.

spectrum of BSA-CuS showed that the absorption peak shifted to a lower wavelength of about $196 \mathrm{~nm}$ after the TAA aqueous solution was added to the $\mathrm{BSA}-\mathrm{Cu}^{2+}$ solution, which indicated that the $\mathrm{S}^{2-}$ released from TAA reacted with $\mathrm{Cu}^{2+}$ to form CuS/BSA nanocomposites. Curve (c) has a gradually increasing absorbance toward shorter wavelengths in accord with the results in the literature [22]. Curve (d) was the spectrum of $\mathrm{CuS}$ formed directly from the reaction between $\mathrm{Cu}^{2+}$ and TAA without BSA, showing broad absorbance at the region of $190-250 \mathrm{~nm}$. This phenomenon is likely due 


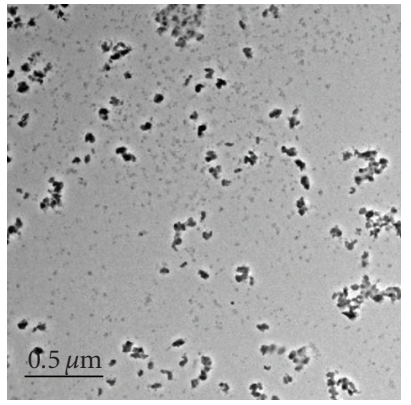

(a)

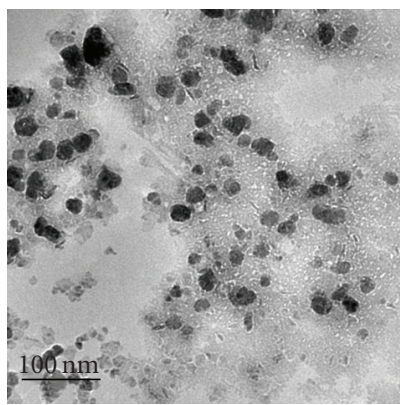

(e)

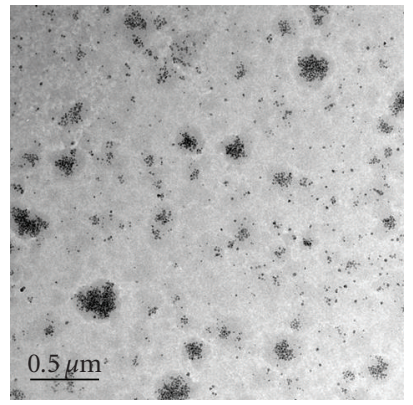

(b)

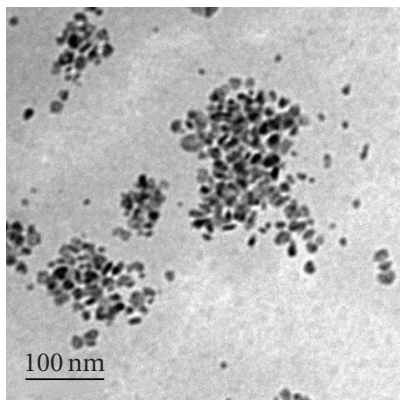

(f)

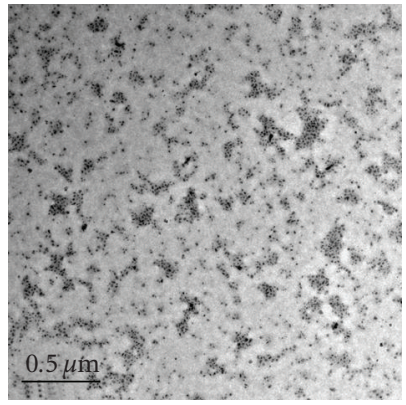

(c)

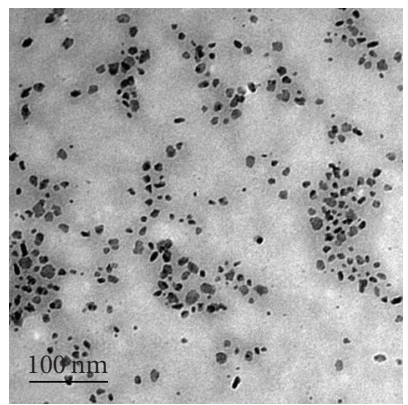

(g)

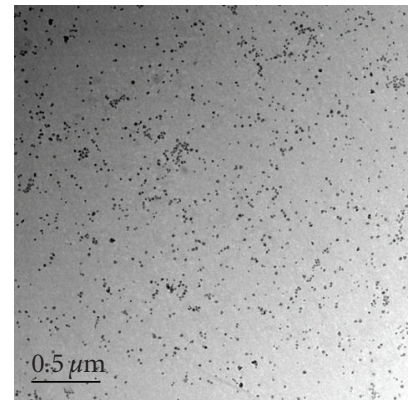

(d)

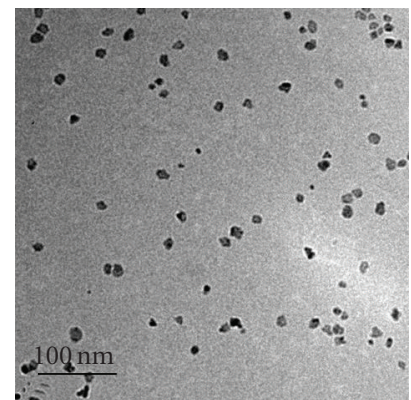

(h)

Figure 3: TEM images ( $a, b, c, d$ ) and the higher magnification TEM images (e, f, g, h) of CuS/BSA nanocomposites in different concentration BSA solution ((a) and (e): $1 \mathrm{mg} / \mathrm{mL}$; (b) and (f): $2 \mathrm{mg} / \mathrm{mL}$; (c) and (g): $3 \mathrm{mg} / \mathrm{mL}$; (d) and (h): $4 \mathrm{mg} / \mathrm{mL}$ ).

to the overlap of absorption bands, which occur at different energies for nanocrystals having disparate sizes.

Figure 2 shows the UV spectra of CuS/BSA nanocomposites synthesized in different concentration BSA solution. All curves indicated that the special absorption peak of CuS/BSA nanocomposites at the same site $(\sim 190 \mathrm{~nm})$. The absorption intensity and half-peak width are both correlated to the particle size of nanomaterials [26, 27]. According to this principle, we can conclude that the size of CuS/BSA nanocomposites is the maximum in BSA $1.0 \mathrm{mg} / \mathrm{mL}$, and the size of CuS/BSA nanocomposites is the minimum in BSA $3.0 \mathrm{mg} / \mathrm{mL}$.

3.2. Morphological Observation. Figure 3 is the TEM images of as-prepared CuS/BSA nanocomposites in different concentration BSA solution, Figures 3(a), 3(b), 3(c), and 3(d) are lower magnification TEM images of CuS/BSA nanocomposites, and Figures $3(\mathrm{e}), 3(\mathrm{f}), 3(\mathrm{~g})$, and $3(\mathrm{~h})$ are the higher magnification TEM images. The CuS/BSA nanocomposites are approximately spherical and the particle size is in the range of $10-35 \mathrm{~nm}$. The formed CuS/BSA nanocomposites exhibited some degree of aggregated morphology in lower concentration BSA solution $(c<2 \mathrm{mg} / \mathrm{mL})$. With the increase of BSA concentration, the monodispersity of $\mathrm{CuS} / \mathrm{BSA}$ nanocomposites was gradually improved, which is attributed to that more and more BSA molecules as stabilizing reagent modify the formed $\mathrm{CuS}$ nanoparticles. Analysis of the TEM images in Figures 3(d) and 3(h) reveals that these spherical nanoparticles are monodispersed with

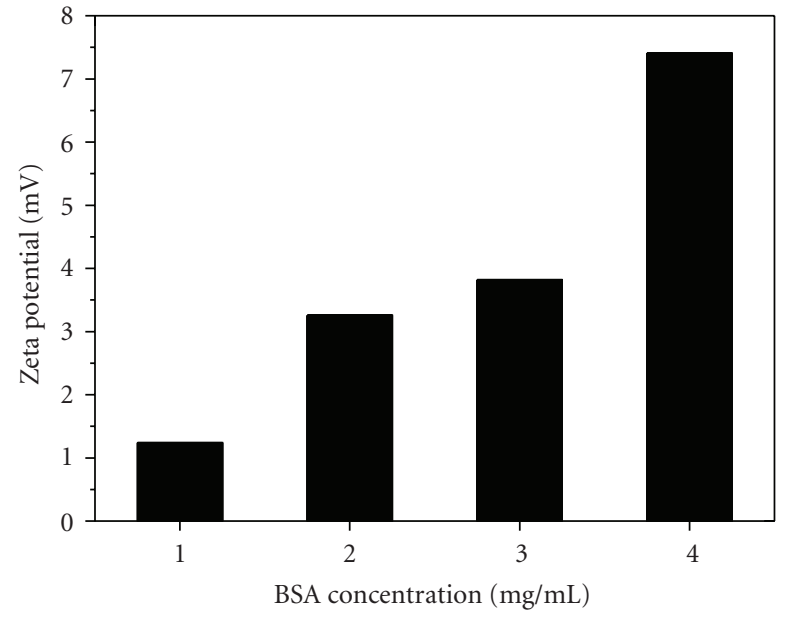

FIGURE 4: Zeta potentials of CuS/BSA nanocomposites in different concentration BSA solution.

average size $16.8 \pm 3.4 \mathrm{~nm}$ by measuring 200 randomly selected particles in enlarged TEM images.

3.3. Zeta Potential Analysis. Zeta potentials of CuS/BSA nanocomposites in different concentration BSA solution were recorded at $\mathrm{pH} 7.0$ as shown in Figure 4. As demonstrated in Figure 4, CuS/BSA nanocomposites all bear positive charge. The value of positive charge was increased 


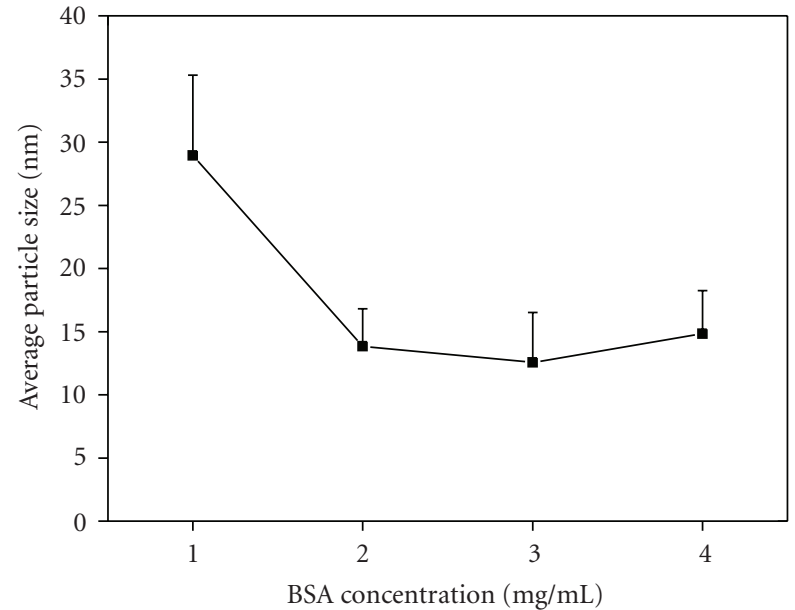

(a)

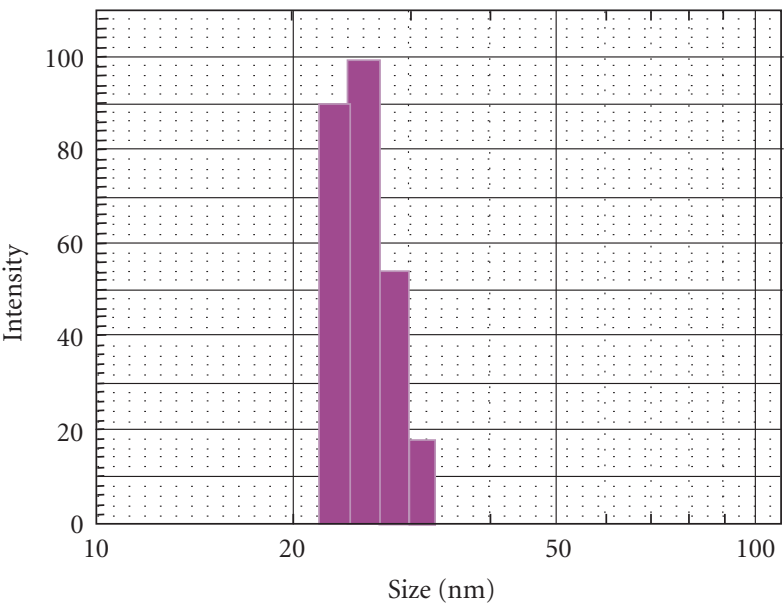

(b)

Figure 5: (a) Average particle size of CuS/BSA nanocomposites in different concentration BSA solution, (b) Dynamic light scattering (DLS) curve of CuS/BSA nanocomposites in $4 \mathrm{mg} / \mathrm{mL}$ BSA solution.

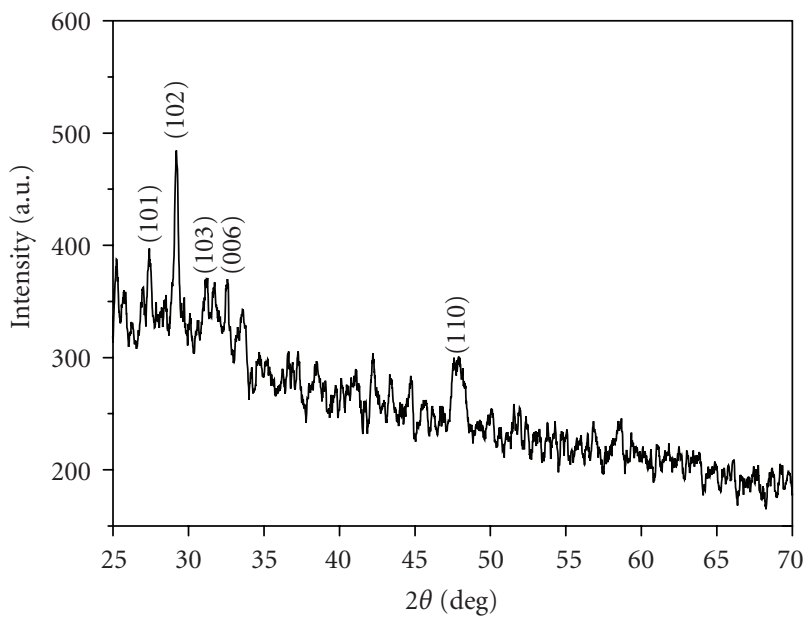

FIgURE 6: XRD pattern of CuS/BSA nanocomposites.

depending highly on concentration of BSA, which is attributed to that the number of BSA molecule without binding $\mathrm{Cu}^{2+}$ was increased in the solution. When BSA concentration was $4 \mathrm{mg} / \mathrm{mL}$, the value of positive charge is the maximum about $+7.41 \mathrm{mV}$, we can deduce that the monodispersity of CuS/BSA nanocomposites at this stage is the best. This result is consistent with TEM images.

3.4. Size Analysis. Figure 5(a) shows that average particle size of CuS/BSA nanocomposites in different concentration BSA solution by measuring 200 randomly selected particles in enlarged TEM images. With the increase of BSA concentration, the size of CuS/BSA nanocomposites gradually decreased. When BSA concentration was over $2 \mathrm{mg} / \mathrm{mL}$, the size was almost not changed. Regarding the potential mechanism, we consider that the number of

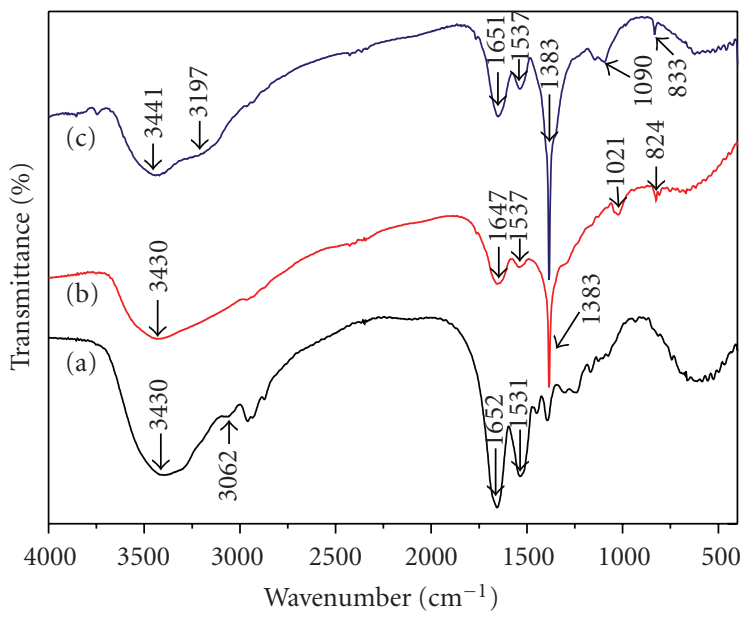

Figure 7: FT-IR spectra of (a) BSA, (b) BSA-Cu ${ }^{2+}$, and (c) BSACuS.

$\mathrm{CuS}$ nucleation depends on the number of binding sites of BSA and $\mathrm{Cu}^{2+}$; the amount of $\mathrm{Cu}^{2+}$, is fixed, so the number of binding sites is also fixed; with the increase of BSA concentration, the binding sites of BSA are also accordingly increased; within the range of concentration of $2 \mathrm{mg} / \mathrm{mL}$ BSA solution, more and more $\mathrm{Cu}^{2+}$ ions were bound to the binding sites of BSA; therefore, the size of CuS/BSA nanocomposites became smaller and smaller; when the BSA concentration reaches over $2 \mathrm{mg} / \mathrm{mL}$, the number of nucleation does not increase; therefore, the size of CuS/BSA nanocomposites is not changed. In addition, the size of CuS/BSA nanocomposites in $4 \mathrm{mg} / \mathrm{mL}$ BSA solution was also determined by dynamic light scattering as shown in Figure 5(b). The average diameter was $25.8 \pm 3.3 \mathrm{~nm}$ which is larger than the result of TEM images analysis. It is ascribed to the BSA molecule on the surface of the 

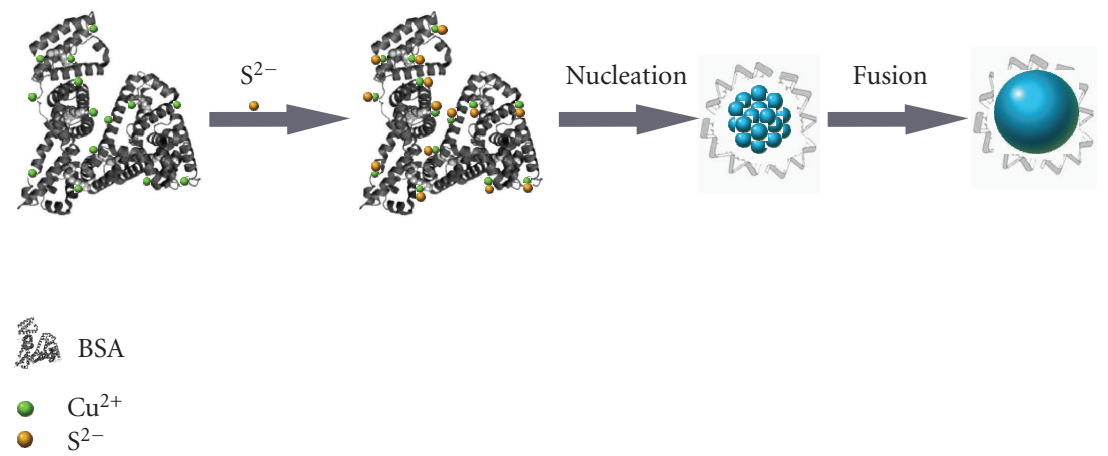

Figure 8: Scheme of CuS/BSA nanocomposites formation in the BSA solution.

nanoparticles, whose average diameter was $\sim 9 \mathrm{~nm}$, and the water molecule around the nanoparticles forming hydrodynamic radius. Subsequently, we also observed that asprepared CuS/BSA nanocomposites were very stable within 6 months. It can be attributed to BSA as a stabilizing agent to block the further growth of the $\mathrm{CuS}$ nanoparticles.

3.5. X-Ray Diffraction. Figure 6 is XRD pattern of asprepared CuS/BSA nanocomposites in the $2 \theta$ range of 25$70^{\circ}$. The characteristic (101), (102), (103), (005), and (110) peaks could be indexed as hexagonal phase $\mathrm{CuS}$ with lattice parameters of $a=3.794 \AA$ and $c=16.35 \AA$, which are consistent with the standard card (JCPDS: 03-1090).

3.6. FT-IR Measurement. To study the formation mechanism of CuS/BSA nanocomposites in the BSA solution, the FTIR spectra of pure BSA, BSA-Cu${ }^{2+}$, and BSA-CuS powders were determined. The FT-IR spectra and the data of the main peaks are, respectively, demonstrated in Figure 7. The IR peaks of pure BSA at 3430, 3062, 1652, and $1531 \mathrm{~cm}^{-1}$ are assigned to the stretching vibration of $\mathrm{OH}$, amide A (mainly - $\mathrm{NH}$ stretching vibration), amide I (mainly $\mathrm{C}=\mathrm{O}$ stretching vibrations), and amide II (the coupling of bending vibrate of $\mathrm{N}-\mathrm{H}$ and stretching vibrate of C-N) bands, respectively. The difference between the IR spectrum of pure BSA and that of $\mathrm{BSA}-\mathrm{Cu}^{2+}$ is obvious such as the characteristic peak of $-\mathrm{NH}$ groups disappearing, suggesting that there might be coordination interaction between $\mathrm{Cu}^{2+}$ and $-\mathrm{NH}$ groups of BSA, which may play an important role in the formation of $\mathrm{CuS}$ nanoparticles. In addition, the new peaks of BSA-Cu${ }^{2+}$ at $1021 \mathrm{~cm}^{-1}$ and $824 \mathrm{~cm}^{-1}$ might be contributed to the interaction of $\mathrm{Cu}^{2+}$ and BSA. The strong peak at $1383 \mathrm{~cm}^{-1}$ in the BSA-Cu${ }^{2+}$ and BSA-CuS spectra is attributed to the absorption of $\mathrm{NO}_{3}{ }^{-1}$, which was introduced by the addition of $\mathrm{Cu}\left(\mathrm{NO}_{3}\right)_{2}$. Comparing the IR spectra of BSA-CuS with those of pure $\mathrm{BSA}$, the characteristic peak of $-\mathrm{OH}$ groups shifts to a high wavenumber of about $11 \mathrm{~cm}^{-1}$ and the characteristic peak of $-\mathrm{NH}$ groups hardly disappears. The results indicate that there might be conjugate bonds between the
$\mathrm{CuS}$ nanoparticles and $-\mathrm{OH}$ groups and $-\mathrm{NH}$ groups of BSA.

3.7. The Possible Mechanism. A possible mechanism for the formation of $\mathrm{CuS} / \mathrm{BSA}$ nanocomposites is suggested as follows:

$$
\begin{gathered}
n \mathrm{Cu}^{2+}+\mathrm{BSA} \longrightarrow \mathrm{Cu}_{n}-\mathrm{BSA}, \\
\mathrm{Cu}^{2+}+\mathrm{S}^{2-} \longrightarrow \mathrm{CuS}, \\
m \mathrm{CuS}+\mathrm{BSA} \longrightarrow(\mathrm{CuS})_{m}-\mathrm{BSA} .
\end{gathered}
$$

With a net reaction,

$$
m \mathrm{Cu}^{2+}+m \mathrm{~S}^{2-}+\mathrm{BSA} \longrightarrow(\mathrm{CuS})_{m}-\mathrm{BSA} .
$$

The scheme of CuS/BSA nanocomposites formation in the BSA solution is illustrated in Figure 8. Firstly, $\mathrm{Cu}^{2+}$ (green ball) forms complex with BSA as shown in (1) when $\mathrm{Cu}\left(\mathrm{NO}_{3}\right)_{2}$ is added to BSA solution. Secondly, when TAA is added into the solution, the $\mathrm{S}^{2-}$ (yellow ball) combines with $\mathrm{Cu}^{2+}$ cooperated by BSA to form CuS (2). Finally, some CuS (small blue ball) absorb on or bond to BSA molecules and form CuS/BSA nanocomposites (big blue ball) as shown in (3). The CuS/BSA nanocomposites of 10 35 nm in diameter depend highly on concentration of BSA concentration, which also prove the formation of CuS/BSA nanocomposites.

BSA protein can provide multiple binding sites for $\mathrm{Cu}^{2+}$, $\mathrm{Cu}^{2+}$ ions can react with BSA, and the binding sites may include the $-\mathrm{NH},-\mathrm{SH},-\mathrm{COOH}$, and $-\mathrm{OH}$ groups. Then, comparatively unstable TAA slowly hydrolyzed to release the $\mathrm{S}^{2-}$ ion, which reacted with $\mathrm{Cu}^{2+}$ ions to form $\mathrm{CuS}$ nuclei surrounding some special sites of BSA. With aging, the growth of CuS nanoparticles started on them. As a soft template, BSA is very stable stabilizing agents, and has a great effect on the monodisperse of nanoparticles by BSA concentration dependent. Therefore, the CuS/BSA nanocomposites were obtained.

\section{Conclusions}

The monodispersed CuS nanoparticles were successfully synthesized by using BSA as the foaming and stabilizing 
reagent. They are approximately spherical, with a size distribution from 10 to $35 \mathrm{~nm}$ in diameter and good dispersibility, depending highly on concentration of BSA. These proteinassisted synthesized CuS nanomaterials have a great potential application in biomedical engineering and microelectronics.

\section{Acknowledgments}

This work is supported by the National Key Basic Research Program (973 project) (2010CB933901), National $863 \mathrm{Hi}$ tech project (2007AA022004), Important National Science \& Technology Specific projects (2009ZX10004-311), National Natural Scientific Fund (no. 20771075 and no. 20803040), Special project for nanotechnology from Shanghai (no. 1052nm04100), New Century Excellent Talent of Ministry of Education of China (NCET-08-0350), and Shanghai Science and Technology Fund (10XD1406100).

\section{References}

[1] H. Wang, J.-R. Zhang, X.-N. Zhao, S. Xu, and J.-J. Zhu, "Preparation of copper monosulfide and nickel monosulfide nanoparticles by sonochemical method," Materials Letters, vol. 55, no. 4, pp. 253-258, 2002.

[2] X. Zhang, Q. Guo, and D. Cui, "Recent advances in nanotechnology applied to biosensors," Sensors, vol. 9, no. 2, pp. 1033 1053, 2009.

[3] H. Li, Y. Zhang, and W. Huang, "Photoactivation of ionexchangeable trititanate nanotubes modified by $\mathrm{MS}(\mathrm{M}=\mathrm{Cd}$, $\mathrm{Zn}$ ) nanoparticles," Nano Biomedicine and Engineering, vol. 1, pp. 32-37, 2009.

[4] D. Cui, Y. Han, Z. Li, et al., "Fluorescent magnetic nanoprobes for in vivo targeted imaging and hyperthermia therapy of prostate cancer," Nano Biomedicine and Engineering, vol. 1, pp. 61-74, 2009.

[5] C. Liu, "Research and development of nanopharmaceuticals in China," Nano Biomedicine and Engineering, vol. 1, pp. 1-18, 2009.

[6] P. Roy and S. K. Srivastava, "Low-temperature synthesis of CuS nanorods by simple wet chemical method," Materials Letters, vol. 61, no. 8-9, pp. 1693-1697, 2007.

[7] Y. Zhang, Z.-P. Qiao, and X.-M. Chen, "Microwave-assisted elemental-direct-reaction route to nanocrystalline copper sulfides $\mathrm{Cu}_{9} \mathrm{~S}_{8}$ and $\mathrm{Cu}_{7} \mathrm{~S}_{4}$," Journal of Solid State Chemistry, vol. 167, no. 1, pp. 249-253, 2002.

[8] R. Cordova, H. Gomez, R. Schrebler, et al., "Electrosynthesis and electrochemical characterization of a thin phase of $\mathrm{Cu}_{x} \mathrm{~S}(x \rightarrow 2)$ on ITO electrode," Langmuir, vol. 18, no. 22, pp. 8647-8654, 2002.

[9] K. Tang, D. Chen, Y. Liu, G. Shen, H. Zheng, and Y. Qian, "Shape-controlled synthesis of copper sulfide nanocrystals via a soft solution route," Journal of Crystal Growth, vol. 263, no. 1-4, pp. 232-236, 2004.

[10] Z. P. Qiao, Y. Xie, J. G. Xu, Y. J. Zhu, and Y. T. Qian, “ $y$ radiation synthesis of the nanocrystalline semiconductors $\mathrm{PbS}$ and CuS," Journal of Colloid and Interface Science, vol. 214, no. 2, pp. 459-461, 1999.

[11] L. Gao, E. Wang, S. Lian, Z. Kang, Y. Lan, and D. Wu, "Microemulsion-directed synthesis of different CuS nanocrystals," Solid State Communications, vol. 130, no. 5, pp. 309-312, 2004.
[12] Q. Lu, F. Gao, and D. Zhao, "One-step synthesis and assembly of copper sulfide nanoparticles to nanowires, nanotubes, and nanovesicles by a simple organic amine-assisted hydrothermal process," Nano Letters, vol. 2, no. 7, pp. 725-728, 2002.

[13] I. P. Parkin, "Solid state metathesis reaction for metal borides, silicides, pnictides and chalcogenides: ionic or elemental pathways," Chemical Society Reviews, vol. 25, no. 3, pp. 199207, 1996.

[14] E. Godočíková, P. Baláž, E. Gock, W. S. Choi, and B. S. Kim, "Mechanochemical synthesis of the nanocrystalline semiconductors in an industrial mill," Powder Technology, vol. 164, no. 3, pp. 147-152, 2006.

[15] R. Nomura, K. Miyawaki, T. Toyosaki, and H. Matsuda, "Preparation of copper sulfide thin layers by a single source MOCVD process," Chemical Vapor Deposition, vol. 2, no. 5, pp. 174-178, 1996.

[16] S.-H. Yu, "Bio-inspired crystal growth by synthetic templates," Topics in Current Chemistry, vol. 271, pp. 79-118, 2006.

[17] M. Reches and E. Gazit, "Casting metal nanowires within discrete self-assembled peptide nanotubes," Science, vol. 300, no. 5619, pp. 625-627, 2003.

[18] L. Yang, H.-J. Wang, H.-Y. Yang, et al., "Shape-controlled synthesis of protein-conjugated silver sulfide nanocrystals and study on the inhibition of tumor cell viability," Chemical Communications, no. 26, pp. 2995-2997, 2008.

[19] D. C. Carter and J. X. Ho, "Structure of serum albumin," Advances in Protein Chemistry, vol. 45, pp. 153-203, 1994.

[20] A. V. Singh, B. M. Bandgar, M. Kasture, B. L. V. Prasad, and M. Sastry, "Synthesis of gold, silver and their alloy nanoparticles using bovine serum albumin as foaming and stabilizing agent," Journal of Materials Chemistry, vol. 15, no. 48, pp. 5115-5121, 2005.

[21] A. V. Singh, R. Patil, M. B. Kasture, W. N. Gade, and B. L. V. Prasad, "Synthesis of Ag-Pt alloy nanoparticles in aqueous bovine serum albumin foam and their cytocompatibility against human gingival fibroblasts," Colloids and Surfaces B, vol. 69, no. 2, pp. 239-245, 2009.

[22] L. Yang, R. Xing, Q. Shen, et al., "Fabrication of proteinconjugated silver sulfide nanorods in the bovine serum albumin solution," Journal of Physical Chemistry B, vol. 110, no. 21, pp. 10534-10539, 2006.

[23] L. Yang, H. Yang, Z. Yang, et al., "Observation of rotatedoriented attachment during the growth of $\mathrm{Ag}_{2} \mathrm{~S}$ nanorods under mediation of protein," Journal of Physical Chemistry B, vol. 112, no. 32, pp. 9795-9801, 2008.

[24] D. Z. Qin, X. M. Ma, L. Yang, L. Zhang, Z. J. Ma, and J. Zhang, "Biomimetic synthesis of HgS nanoparticles in the bovine serum albumin solution," Journal of Nanoparticle Research, vol. 10, no. 4, pp. 559-566, 2008.

[25] P. Huang, Y. Kong, Z. Li, F. Gao, and D. Cui, “Copper selenide nanosnakes: bovine serum albumin-assisted room temperature controllable synthesis and characterization," Nanoscale Research Letters, 2010.

[26] P. Huang, J. Lin, Z. Li, et al., Chemical Communications, 2010, in press.

[27] H. Hu, H. Yang, P. Huang, et al., "Unique role of ionic liquid in microwave-assisted synthesis of monodisperse magnetite nanoparticles," Chemical Communications, 2010. 

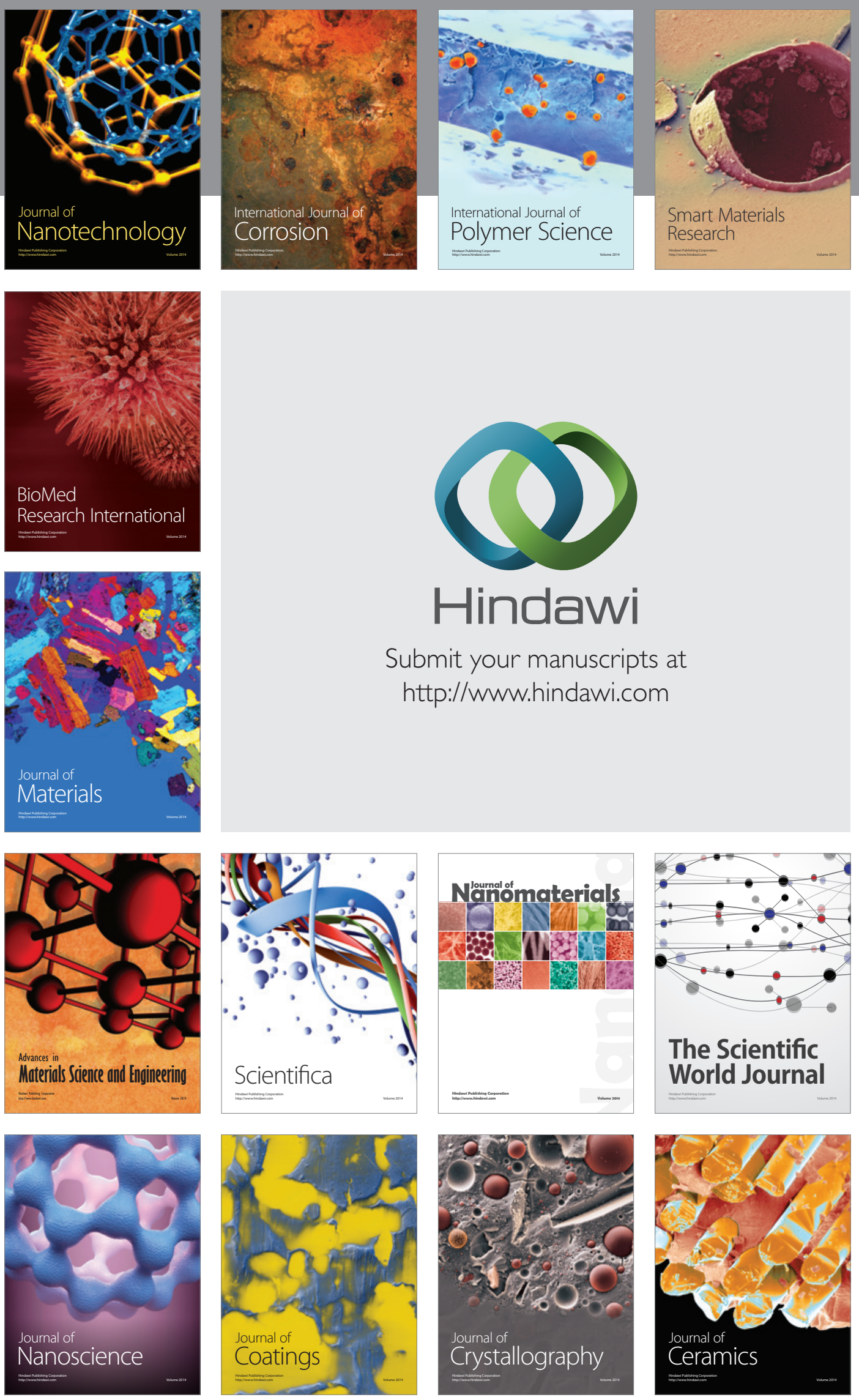

The Scientific World Journal

Submit your manuscripts at

http://www.hindawi.com

\section{World Journal}

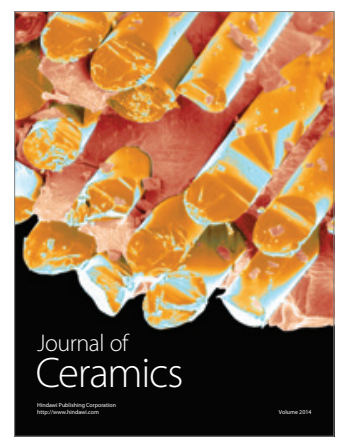

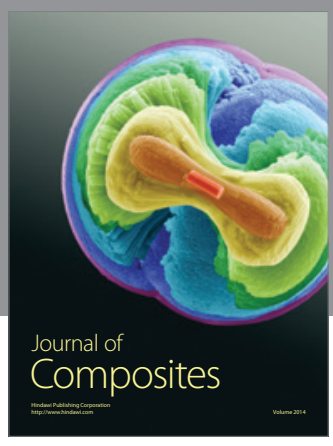
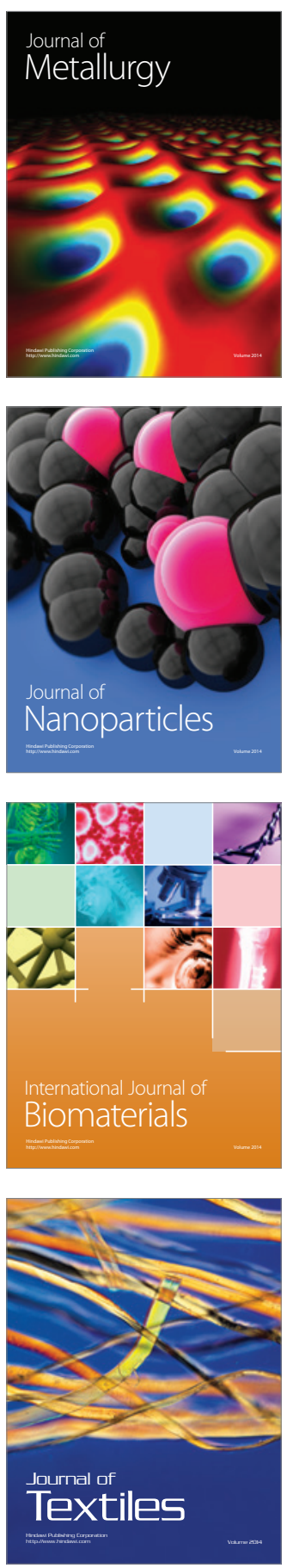